\title{
Parity numerical semigroups
}

\author{
María Ángeles Moreno-Frías ${ }^{* 1}$ (D), José Carlos Rosales ${ }^{2}$ (D) \\ ${ }^{1}$ Dpto. Matemáticas, Facultad de Ciencias, Universidad de Cádiz, E-11510, Puerto Real, Cádiz, Spain \\ ${ }^{2}$ Dpto. de Álgebra, Facultad de Ciencias, Universidad de Granada, E-18071, Granada, Spain
}

\begin{abstract}
A numerical semigroup is parity (P-semigroup) if the sum of two consecutive elements of $S$ is odd. In this paper we will order the $\mathrm{P}$-semigroups creating a tree with root. This ordering allows us to give some algorithms to build all the P-semigroups with a fixed genus and Frobenius number. Also, we will study the maximal P-semigroups with a given Frobenius number as well as the P-semigroups with maximum embedding dimension.
\end{abstract}

Mathematics Subject Classification (2010). 20M14, 11D07

Keywords. embedding dimension, Frobenius number, genus, multiplicity, numerical semigroup

\section{Introduction}

We denote by $\mathbb{Z}$ and $\mathbb{N}$ the set of integers numbers and the nonnegative integers numbers, respectively. A numerical semigroup is a subset $S$ of $\mathbb{N}$ which is closed by sum, $0 \in S$ and $\mathbb{N} \backslash S$ is finite.

If $S$ is a numerical semigroup, then $\mathrm{m}(S)=\min (S \backslash\{0\}), \mathrm{F}(S)=\max (\mathbb{Z} \backslash S)$ and $\mathrm{g}(S)=$ $\sharp(\mathbb{N} \backslash S)$, where $\sharp(A)$ denote the cardinality of a set $A$, they are three important invariants of $S$ which we will call multiplicity, Frobenius number and genus of $S$, respectively.

If $S$ is a numerical semigroup and $x \in S$, we denote by $\operatorname{next}_{S}(x)=\min \{s \in S \mid x<s\}$ and $\operatorname{prev}_{S}(x)=\max \{s \in S \mid s<x\}$. A numerical semigroup is parity (P-semigroup) if $x+\operatorname{next}_{S}(x)$ is odd for every $x \in S$. We say that a sequence is a parity sequence if the sum of two consecutive elements is odd.

Our main goal in this paper is to begin the study of the parity numerical semigroups. These semigroups are a distinguished class within the so-called perfect numerical semigroups introduced in [2]. Indeed, a numerical semigroup is perfect if $\{x-1, x+1\} \subseteq S$ implies $x \in S$. It is clear then that every parity numerical semigroup is a perfect numerical semigroup.

In Section 2, we will order the elements of $\mathcal{P}_{m}=\{S \mid S$ is a P-semigroup and $m(S)=$ $m$ \} making a tree with root. We will characterize the children of a vertex and this will allow us to build recursively the elements of $\mathcal{P}_{m}$. These results will be used in Section 3 in order to show some algorithms which enable us to compute all the P-semigroups with fixed genus and fixed Frobenius number.

\footnotetext{
*Corresponding Author.

Email addresses: mariangeles.moreno@uca.es (M. A. Moreno-Frías), jrosales@ugr.es (J. C. Rosales)

Received: 02.04.2019; Accepted: 19.07.2019
} 
In Section 4, we are interested in studying the P-semigroups which are maximal in the set of all semigroups with fixed Frobenius number. In particular, we will give a construction that permits to add elements to a $\mathrm{P}$-semigroup with Frobenius number $\mathrm{F}$ until it turns into a maximal P-semigroup with Frobenius number F.

Finally, in Section 5 we will study the P-semigroups with maximun embedding dimension. In particular, we will show a construction that allows us to compute from a Psemigroup and one of its odd elements, another a P-semigroup with maximun embedding dimension.

\section{The tree of the P-semigroups}

Let $S$ be a P-semigroup with multiplicity $m$. Then $0+\operatorname{next}_{S}(0)=0+m$ is odd. Therefore the multiplicity of a P-semigroup is always an odd number. In addition, if $m$ is an odd, nonnegative integer then the set $\{0, m, \rightarrow\}$ (the symbol $\rightarrow$ means that every integer greater than $m$ belongs to the set), is a P-semigroup. Therefore we have the following result.

Lemma 2.1. $\mathcal{P}_{m} \neq \emptyset$ if and only if $m$ is an odd positive integer.

It is clear that if $m$ is an odd positive integer greater than or equal to 3 and $k \in \mathbb{N} \backslash\{0\}$ then $\Delta(k)=\{0, m, 2 m, \ldots, k m, \rightarrow\} \in \mathcal{P}_{m}$. Hence, if $\mathcal{P}_{m} \neq \emptyset$ and $m \geq 3$ then $\mathcal{P}_{m}$ has infinity cardinality.

Lemma 2.2. If $S$ is a P-semigroup then $\mathrm{F}(S)-1 \notin S$.

Proof. If $\mathrm{F}(S)-1 \in S$, then $\operatorname{next}_{S}(\mathrm{~F}(S)-1)=\mathrm{F}(S)+1$. Therefore, $\mathrm{F}(S)-1+\operatorname{next}_{S}(\mathrm{~F}(S)-$ $1)=2 \mathrm{~F}(S)$ is an even number which is against the fact that $\mathrm{S}$ is a $\mathrm{P}$-semigroup.

Note that if $m$ is an odd positive integer then the set $\{0, m, \rightarrow\}$ is the maximun (with respect to inclusion order) of $\mathcal{P}_{m}$.

Lemma 2.3. Let $m$ be an odd integer greater than or equal to 3 and $S \in \mathcal{P}_{m}$ such that $S \neq\{0, m, \rightarrow\}$. Then $T=S \cup\{\mathrm{F}(S), \mathrm{F}(S)-1\} \in \mathcal{P}_{m}$.

Proof. It is clear that $T$ is a numerical semigroup. Since $\operatorname{prev}_{S}(\mathrm{~F}(S)+1)=\operatorname{prev}_{T}(\mathrm{~F}(S)-$ 1) and $S \in \mathcal{P}_{m}$, it follows that $\operatorname{prev}_{T}(\mathrm{~F}(S)-1)+(\mathrm{F}(S)-1)=\operatorname{prev}_{S}(\mathrm{~F}(S)+1)+(\mathrm{F}(S)+1)-2$ is odd. Since both $2 \mathrm{~F}(S) \mp 1$ are odd, $T \in \mathcal{P}_{m}$.

The above lemma allows us to define recurrently the following sequence of elements of $\mathcal{P}_{m}$. If $S \in \mathcal{P}_{m}$ then:

- $S_{0}=S$,

- $S_{n+1}= \begin{cases}S_{n} \cup\left\{\mathrm{F}\left(S_{n}\right), \mathrm{F}\left(S_{n}\right)-1\right\} & \text { if } S_{n} \neq\{0, m, \rightarrow\}, \\ S_{n} & \text { otherwise. }\end{cases}$

The following result can be easily tested.

Lemma 2.4. If $S \in \mathcal{P}_{m}$ then there exist $k \in \mathbb{N}$ and $S_{0}, S_{1}, \ldots, S_{k} \in \mathcal{P}_{m}$ such that $S=$ $S_{0} \subsetneq S_{1} \subsetneq \ldots \subsetneq S_{k}=\{0, m, \rightarrow\}$. In addition $\sharp\left(S_{i+1} \backslash S_{i}\right)=2$ for every $i \in\{0, \ldots, k-1\}$ and $k=\frac{\mathrm{g}(S)-(m-1)}{2}$.

We illustrate the above result with an example.

Example 2.5. It is clear that $S=\{0,7,10,13,14,15,16,17,20, \rightarrow\} \in \mathcal{P}_{7}$. Then we have the following sequence of elements of $\mathcal{P}_{7}$ :

$$
\begin{gathered}
S_{0}=\{0,7,10,13,14,15,16,17,20, \rightarrow\} \subsetneq S_{1}=\{0,7,10,13, \rightarrow\} \subsetneq \\
S_{2}=\{0,7,10, \rightarrow\} \subsetneq S_{3}=\{0,7, \rightarrow\} .
\end{gathered}
$$

Remark that as an immediate consequence of Lemma 2.4 we have the following result. 
Lemma 2.6. If $S$ is a P-semigroup then $g(S)$ is even. Moreover, for any $x, y \in S$ with $x<y$ if $S \cap[x+1, y-1]=\emptyset$ then $\sharp\{x+1, \ldots, y-1\}$ is even.

A graph $G$ is a pair $(V, E)$ where $V$ is a not empty set and $E$ is a subset of $\{(u, v) \in$ $V \times V \mid u \neq v\}$. The elements of $V$ and $E$ are called vertices and edges respectively.

A path (of length $n$ ) connecting the vertices $x$ and $y$ of $G$ is a sequence of different edges of the form $\left(v_{0}, v_{1}\right),\left(v_{1}, v_{2}\right), \ldots,\left(v_{n-1}, v_{n}\right)$ such that $v_{0}=x$ and $v_{n}=y$.

A graph $G$ is a tree if there exists a vertex $r$ (known as the root of $G$ ) such that for any other vertex $x$ of $G$ there exists a unique path connecting $x$ and $r$. If there exists a path connecting the vertices $x$ and $y$, then we will call that $x$ is a descendant of $y$. In particular, if $(x, y)$ is an edge of the tree, we say that $x$ is a child of $y$.

We define the graph $G\left(\mathcal{P}_{m}\right)$ as follows: $\mathcal{P}_{m}$ is its set of vertices and $(S, T) \in \mathcal{P}_{m} \times \mathcal{P}_{m}$ is an edge if $T=S \cup\{\mathrm{F}(S), \mathrm{F}(S)-1\}$.

The following result is a consequence of Lemma 2.4 .

Proposition 2.7. If $m$ is an odd positive integer, then $G\left(\mathcal{P}_{m}\right)$ is a tree whose root is the $P$-semigroup $\{0, m, \rightarrow\}$.

If $A$ is a not empty subset of $\mathbb{N}$, we denote by $\langle A\rangle$ the submonoid of $(\mathbb{N},+)$ generated by $A$, that is $\langle A\rangle=\left\{\lambda_{1} a_{1}+\cdots+\lambda_{n} a_{n} \mid n \in \mathbb{N}, a_{1}, \ldots, a_{n} \in A\right.$ and $\left.\lambda_{1}, \ldots, \lambda_{n} \in \mathbb{N}\right\}$. It is well known that $\langle A\rangle$ is a numerical semigroup if and only if $\operatorname{gcd}(A)=1$ (see for instance [4, Lemma 2.1]).

If $S$ is a numerical semigroup and $S=\langle A\rangle$, then we say that $A$ is a system of generators of $S$. Moreover, if $S \neq\langle B\rangle$ for every $B \subsetneq A$ then we say that $A$ is a minimal system of generators of $S$. In [4, Corollary 2.8], it is shown that every numerical semigroup has a unique minimal system of generators and this system is finite. We denote by $\operatorname{msg}(S)$ the minimal system of generators of $S$; its cardinality is called the embedding dimension of $S$ and it is denoted by $e(S)$. Also it is well known (see [4, Exercise 2.1]), the following result.

Lemma 2.8. Let $S$ be a numerical semigroup and $x \in S$. Then $S \backslash\{x\}$ is a numerical semigroup if and only if $x \in \operatorname{msg}(S)$.

A tree can be built recurrently starting from its root and adding to each vertex already built, its children. In the following result we are going to see who are the children of a vertex of $G\left(\mathcal{P}_{m}\right)$.

Proposition 2.9. Let $m$ be an odd integer greater than or equal to 3 and $T \in \mathcal{P}_{m}$. Then $S$ is a child of $T$ in the tree $G\left(\mathcal{P}_{m}\right)$ if and only if $S=T \backslash\{x, x+1\}$ and $\{x, x+1\} \subseteq\{a \in$ $\operatorname{msg}(T) \mid a>\mathrm{F}(T)$ and $a \neq m\}$.

Proof. Necessity. $T=S \cup\{\mathrm{F}(S)-1, \mathrm{~F}(S)\}$, then it is clear that $\{\mathrm{F}(S)-1, \mathrm{~F}(S)\} \subseteq\{a \in$ $\operatorname{msg}(T) \mid a>\mathrm{F}(T)$ and $m \neq \mathrm{F}(S)-1\}$.

Sufficiency. It is clear, using Lemma 2.8, that $S=T \backslash\{x, x+1\}$ is a P-semigroup with multiplicity $m$ and Frobenius number $x+1$. Hence, $T=S \cup\{\mathrm{F}(S), \mathrm{F}(S)-1\}$ and whence $S$ is child of $T$.

Now, we illustrate the before results with an example. 
Example 2.10. We are going to build the tree $G\left(\mathcal{P}_{5}\right)$ :

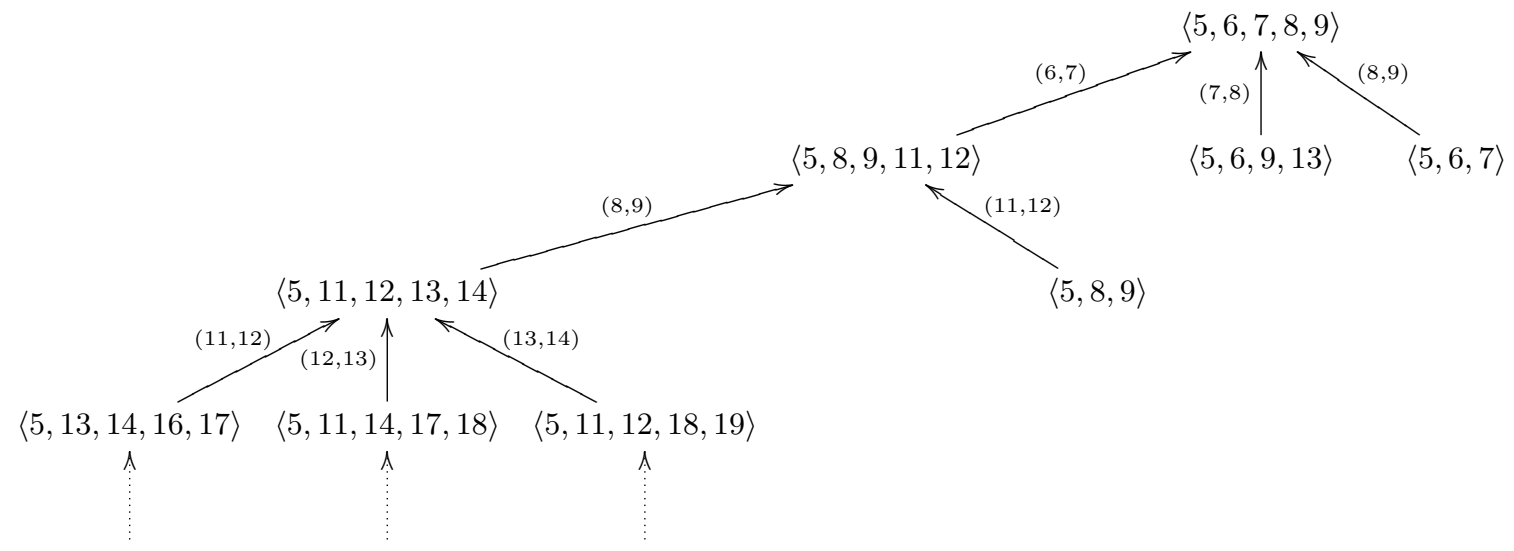

The pair that appears on the edge indicates the minimal generators that we eliminate from T. Therefore, if on the edge $(S, T)$ appears the pair $(x, x+1)$ then $S=T \backslash\{x, x+1\}$. Note that in this case $\mathrm{F}(S)=x+1$.

\section{The P-semigroups with fixed Frobenius number and fixed genus}

If $G=(V, E)$ is a tree and $x$ is a vertex of $G$, then the depth of $x$, that we will denote by $d(x)$, is the length of a unique path that connects $x$ with the root. If $k \in \mathbb{N}$ we denote by $N(G, k)=\{x \in V \mid d(x)=k\}$. The height of $G$, that we will denote by $h(G)$, is the maximun of the set $\{k \in \mathbb{N} \mid N(G, k) \neq \emptyset\}$.

Using Lemma 2.4, it is easy to prove the following result.

Lemma 3.1. Let $m$ be an odd integer greater than or equal to 3 and $S \in \mathcal{P}_{m}$. Then $S \in N\left(G\left(\mathcal{P}_{m}\right), k\right)$ if and only if $\mathrm{g}(S)=m-1+2 k$.

We are now in a position to give an algorithm that calculates us all the P-semigroups with fixed multiplicity and fixed genus. Note that if $S$ is a numerical semigroup then $\mathrm{g}(S) \geq \mathrm{m}(S)-1$. Remember also that by Lemma 2.6 we know that if $S$ is a P-semigroup then $\mathrm{g}(S)$ is even.

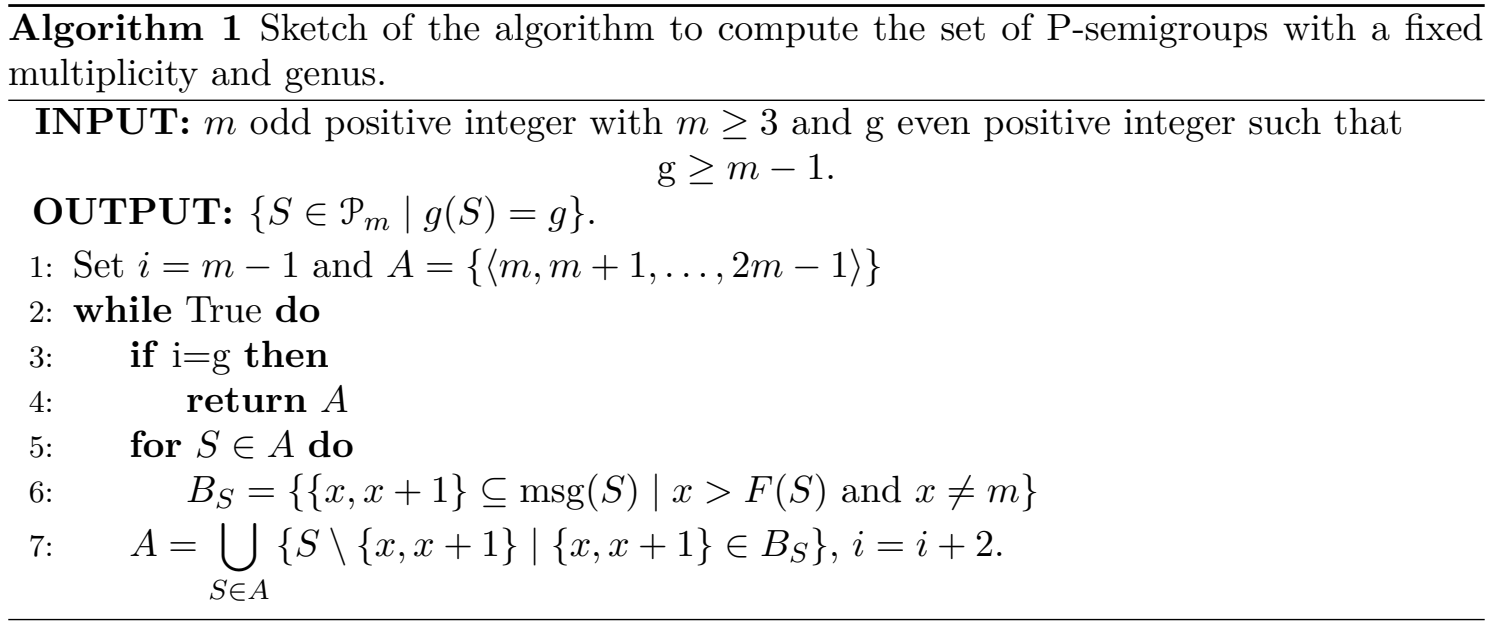

Now we will illustrate how the previous algorithm works with an example. 
Example 3.2. We are going to compute all the P-semigroups with multiplicity 5 and genus 8. For this we will use the Algorithm 1:

- $A=\{\langle 5,6,7,8,9\rangle\}, i=4$

- $B_{\langle 5,6,7,8,9\rangle}=\{\{6,7\},\{7,8\},\{8,9\}\}$

- $A=\{\langle 5,8,9,11,12\rangle,\langle 5,6,9,13\rangle,\langle 5,6,7\rangle\}, i=6$

- $B_{\langle 5,8,9,11,12\rangle}=\{\{8,9\},\{11,12\}\}, B_{\langle 5,6,9,13\rangle}=\emptyset, B_{\langle 5,6,7\rangle}=\emptyset$

- $A=\{\langle 5,11,12,13,14\rangle,\langle 5,8,9\rangle\}, i=8$

- $\left\{S \in \mathcal{P}_{5} \mid \mathrm{g}(S)=8\right\}=\{\langle 5,11,12,13,14\rangle,\langle 5,8,9\rangle\}$.

Given two integers $a$ and $b$ with $b \neq 0$, we denote by $a \bmod b$ the remainder of the division of $a$ by $b$. Let $m$ and $\mathrm{F}$ be positive integers. Our next aim in this section will be to show an algorithm that allows us to compute the set $\left\{S \in \mathcal{P}_{m} \mid \mathrm{F}(S)=\mathrm{F}\right\}$.

Proposition 3.3. Let $m$ and $\mathrm{F}$ be positive integers. The following conditions are equivalent:

1) $\left\{S \in \mathcal{P}_{m} \mid \mathrm{F}(S)=\mathrm{F}\right\} \neq \emptyset$.

2) $\mathrm{F} \geq m-1 \geq 2, m$ odd and $\mathrm{F} \bmod m \notin\{0,1\}$.

Proof. 1) implies 2). Let $S \in \mathcal{P}_{m}$ such that $\mathrm{F}(S)=\mathrm{F}$. As $\mathcal{P}_{m} \neq \emptyset$, then we know, by Lemma 2.1, that $m$ is odd. As $m-1 \notin S$ then $\mathrm{F} \geq m-1 \geq 2$. If $\mathrm{F} \bmod m=0$ then $\mathrm{F} \in\langle m\rangle \subset S$, which makes no sense. If $\mathrm{F} \bmod m=1$ then $\mathrm{F}-1 \in\langle m\rangle \subseteq S$, contradicting Lemma 2.2

2) implies 1). If $\mathrm{F}=m-1$ then $\{0, m, \rightarrow\} \in\left\{S \in \mathcal{P}_{m} \mid \mathrm{F}(S)=\mathrm{F}\right\}$. Therefore, we will assume that $\mathrm{F}>m$, whence $\mathrm{F}=q m+r$ with $q \in \mathbb{N} \backslash\{0\}$ and $r=\mathrm{F} \bmod m$. We distinguish two cases:

(1) If $r$ is even not zero, then $\{0, m, 2 m, \ldots, q m, q m+r+1, \rightarrow\}$ is an element of $\mathcal{P}_{m}$ with Frobenius number F.

(2) If $r$ is odd different from one, then $\{0, m, 2 m, \ldots, q m, q m+1, q m+r+1, \rightarrow\}$ is an element of $\mathcal{P}_{m}$ with Frobenius number F.

We are already able to give the announced algorithm.

$\overline{\text { Algorithm } 2 \text { Sketch of the algorithm to compute the set of all P-semigroup with a fixed }}$ multiplicity and Frobenius number.

INPUT: Integers $m$ and $\mathrm{F}$ such that $m$ is odd, $\mathrm{F}>m \geq 3$ and $\mathrm{F} \bmod m \notin\{0,1\}$.

OUTPUT: $\left\{S \in \mathcal{P}_{m} \mid \mathrm{F}(S)=\mathrm{F}\right\}$.

1: $B=\emptyset$ and $A=\{\langle m, m+1, \ldots, 2 m-1\rangle\}$

2: while True do

3: $\quad$ for $S \in A$ do

4: $\quad$ Compute $B_{S}=\{\{x, x+1\} \subseteq \operatorname{msg}(S) \mid x \neq m, x>F(S)$ and $x+1 \leq F\}$

5: $\quad B=B \cup\left\{S \backslash\{x, x+1\} \mid S \in A,\{x, x+1\} \in B_{S}\right.$ and $\left.x+1=F\right\}$

6: $\quad A=\bigcup_{S \in A}\left\{S \backslash\{x, x+1\} \mid\{x, x+1\} \in B_{S}\right.$ and $\left.x+1<F\right\}$

7: $\quad$ if $A=\emptyset$ then

8: $\quad$ return $B$

Next we illustrate this algorithm with an example.

Example 3.4. We are going to compute all the P-semigroups with multiplicity 5 and Frobenius number 12. For this we will use the Algorithm 2:

- $B=\emptyset$ and $A=\{\langle 5,6,7,8,9\rangle\}$

- $B_{\langle 5,6,7,8,9\rangle}=\{\{6,7\},\{7,8\},\{8,9\}\}$

- $B=\emptyset$ 
- $A=\{\langle 5,8,9,11,12\rangle,\langle 5,6,9,13\rangle,\langle 5,6,7\rangle\}$

- $B_{\langle 5,8,9,11,12\rangle}=\{\{8,9\},\{11,12\}\}, B_{\langle 5,6,9,13\rangle}=\emptyset, B_{\langle 5,6,7\rangle}=\emptyset$

- $B=\{\langle 5,8,9\rangle\}$

- $A=\{\langle 5,11,12,13,14\rangle\}$

- $B_{\langle 5,11,12,13,14\rangle}=\{\{11,12\}\}$

- $B=\{\langle 5,8,9\rangle,\langle 5,13,14,16,17\rangle\}$

- $A=\emptyset$

- $\left\{S \in \mathcal{P}_{5} \mid \mathrm{F}(S)=12\right\}=\{\langle 5,8,9\rangle,\langle 5,13,14,16,17\rangle\}$.

Our next goal in this section will be to show explicitly the minimal elements (with respect to inclusion order) of the set $\left\{S \in \mathcal{P}_{m} \mid \mathrm{F}(S)=\mathrm{F}\right\}$. We will distinguish two cases depending on whether $\mathrm{F}$ mod $m$ is odd or even.

Proposition 3.5. Let $m$ and $\mathrm{F}$ be integers such that $\mathrm{F}>m \geq 3, m$ odd and $\mathrm{F} \bmod m$ an even number not zero. Then $\langle m\rangle \cup\{\mathrm{F}+1, \rightarrow\}=\min \left\{S \in \mathcal{P}_{m} \mid \mathrm{F}(S)=\mathrm{F}\right\}$.

Proof. From the proof of the Proposition 3.3 we know that $\langle m\rangle \cup\{\mathrm{F}+1, \rightarrow\} \in\left\{S \in \mathcal{P}_{m} \mid\right.$ $\mathrm{F}(S)=\mathrm{F}\}$. The proof is finished by the fact that if $S \in \mathcal{P}_{m}$ and $\mathrm{F}(S)=\mathrm{F}$ then clearly $\langle m\rangle \cup\{\mathrm{F}+1, \rightarrow\} \subseteq S$.

Next, we are going to study the case where $\mathrm{F} \bmod m$ is an odd integer different from 1 . Note that if $m=3$ and $\mathrm{F} \bmod m$ is odd then $\mathrm{F} \bmod m=1$. This justifies the fact that in the following proposition, we suppose $m \geq 5$.

Proposition 3.6. Let $m \geq 5$ be an odd integer and $F=q m+2 k+1$ where $q \in \mathbb{Z}^{+}$and $1 \leq k \leq \frac{m-3}{2}$. Then for every $0 \leq i \leq k-1$,

$$
S(i)=\langle m\rangle \cup\{q m+2 i+1, F+1, \rightarrow\}
$$

is a minimal element of $\left\{S \in \mathcal{P}_{m} \mid \mathrm{F}(S)=\mathrm{F}\right\}$.

Proof. It is clear that $S(i)=\{0, m, 2 m, \ldots, q m, q m+2 i+1, q m+2 k+2, \rightarrow\}$. Then we easily deduce that $S(i) \in\left\{S \in \mathcal{P}_{m} \mid \mathrm{F}(S)=F\right\}$ for every $0 \leq i \leq k-1$.

If $S \in \mathcal{P}_{m}$ and $\mathrm{F}(S)=\mathrm{F}$, then $\{0, m, \ldots, q m, \mathrm{~F}+1, \rightarrow\} \subseteq S$. As $q m+\mathrm{F}+1=$ $q m+q m+2 k+2$ is even and $S$ is a P-semigroup, then there exists $s \in S$ such that $q m<s<F+1$ and $q m+s$ is odd. So, we can easily deduce that $s=q m+2 i+1$ for some $i \in\{0, \ldots, k-1\}$. Hence $S(i) \subseteq S$.

Therefore we conclude

$$
\{S(i) \mid i \in\{0, \ldots, k-1\}\}=\operatorname{minimals}_{\subseteq}\left\{S \in \mathcal{P}_{m} \mid \mathrm{F}(S)=\mathrm{F}\right\} .
$$

Note that in the conditions of Proposition 3.6, if $m=5$ then $k=1$ and $i=0$. Hence $S(0)=\langle m\rangle \cup\{q m+1, F+1, \rightarrow\}=\min \left\{S \in \mathcal{P}_{m} \mid \mathrm{F}(S)=\mathrm{F}\right\}$. If $m \geq 7$ and $F=q m+3$ then $\langle m\rangle \cup\{q m+1, q m+4, \rightarrow\}=\min \left\{S \in \mathcal{P}_{m} \mid \mathrm{F}(S)=q m+3\right\}$. If $m \geq 7$ and $F \bmod m$ is an odd integer greater than or equal to 5 then $\left\{S \in \mathcal{P}_{m} \mid F(S)=F\right\}$ has not minimum and it has at least two minimal elements.

Example 3.7. We have that

- By Proposition 3.5, we know that $S=\langle 7\rangle \cup\{19, \rightarrow\}=\{0,7,14,19, \rightarrow\}$ is the minimun of the set $\left\{S \in \mathcal{P}_{7} \mid \mathrm{F}(S)=18\right\}$.

- For the previous comment to this example, we know that $S=\langle 7\rangle \cup\{15,18, \rightarrow\}=$ $\{0,7,14,15,18, \rightarrow\}=\min \left\{S \in \mathcal{P}_{7} \mid \mathrm{F}(S)=17\right\}$.

- By Proposition 3.6, we know that $S(0)=\langle 7\rangle \cup\{15,20, \rightarrow\}=\{0,7,14,15,20, \rightarrow\}$ and $S(1)=\langle 7\rangle \cup\{17,20, \rightarrow\}=\{0,7,14,17,20, \rightarrow\}$ are the minimal elements of $\left\{S \in \mathcal{P}_{7} \mid \mathrm{F}(S)=19\right\}$. 


\section{The maximal P-semigroups with fixed Frobenius number}

In this section we are interested in studying the $\mathrm{P}$-semigroups that are maximal in the set of all $\mathrm{P}$-semigroups which have a fixed Frobenius number.

Lemma 4.1 ([5, Lemma 10]). Let $S$ and $T$ be two numerical semigroups such that $S \subsetneq T$. Then $S \cup\{\max (T \backslash S)\}$ is also a numerical semigroup.

Remark that the previous lemma is not true if we replace numerical semigroup by P-semigroup. It is enough to note that by Lemma 2.6 all the P-semigroups have even genus.

Proposition 4.2. Let $S$ and $T$ be two P-semigroups with $S \subsetneq T$. If $x=\max (T \backslash S)$ then $\operatorname{prev}_{T}(x) \notin S$.

Proof. First of all notice that $\operatorname{prev}_{T}(x)+\operatorname{next}_{T}(x)$ is an even number. Then let $y=$ $\max \{s \in S \mid s<x\}$ which is clearly exists. Assume that $y=\operatorname{prev}_{T}(x)$. Since $\operatorname{next}_{S}(y)=$ $\operatorname{next}_{T}(x)$, it follows that $y+\operatorname{next}_{S}(y)=y+\operatorname{next}_{T}(x)=\operatorname{prev}_{T}(x)+\operatorname{next}_{T}(x)$ is even which

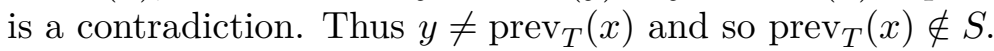

As an immediate consequence of the above proposition, we have the following result.

Corollary 4.3. Let $S$ and $T$ be two P-semigroups such that $S \subsetneq T, x=\max (T \backslash S)$ and $y=\max (T \backslash(S \cup\{x\}))$. Then $S \cup\{x, y\}$ is a P-semigroup.

Now we illustrate the previous results with an example.

Example 4.4. (1) It is clear that $S=\langle 5,11,14,17,18\rangle$ and $T=\langle 5,8,9,11,12\rangle$ are two P-semigroups and $S \subsetneq T$. Moreover $13=\max (T \backslash S)$ and $12=\max (T \backslash(S \cup$ $\{13\}))$. Then by Corollary 4.3 , we have that $S \cup\{12,13\}=\langle 5,11,12,13,14\rangle$ is a P-semigroup.

(2) It is clear that $S=\{0,11,22,33, \rightarrow\}$ and $T=\{0,11,22,23,24,25,32, \rightarrow\}$ are Psemigroups and $S \subsetneq T$. Furthermore, $32=\max (T \backslash S)$ and $25=\max (T \backslash(S \cup$ $\{32\})$ ). Applying Corollary 4.3, we have that $S \cup\{32,25\}$ is a P-semigroup.

Let $S$ be a numerical semigroup. An element $a \in S$ is called refinable if there exists $\{x, y\} \subseteq\left\{b \in \mathbb{N} \mid a<b<\operatorname{next}_{S}(a)\right\}$ such that $\mathrm{F}(S)>x>y, S \cup\{x, y\}$ is a numerical semigroup and $a, y, x, \operatorname{next}_{S}(a)$ is a parity sequence. Denote by $\mathcal{R}(S)=\{a \in S \mid a$ is refinable $\}$.

Corollary 4.5. Let $S$ be a P-semigroup with Frobenius number F. Then the following conditions are equivalent:

1) $S$ is a maximal element in the set $\{T \mid T$ is P-semigroup and $\mathrm{F}(T)=\mathrm{F}\}$.

2) $\mathcal{R}(S)=\emptyset$.

Proof. 1) implies 2). If $\mathcal{R}(S) \neq \emptyset$ then there exists $a \in \mathcal{R}(S)$. Let $\{x, y\} \subseteq\{b \in$ $\left.\mathbb{N} \mid a<b<\operatorname{next}_{S}(a)\right\}$ such that $\mathrm{F}(S)>x>y, S \cup\{x, y\}$ is a numerical semigroup and $a, y, x, \operatorname{next}_{S}(a)$ is a parity sequence. Then it is clear that $S \cup\{x, y\} \in\{T \mid$ $T$ is P-semigroup and $\mathrm{F}(T)=F\}$ and $S \subsetneq S \cup\{x, y\}$ contradicting the maximality of $S$. 2) implies 1). We suppose now that $S$ is not maximal. Therefore there exists a Psemigroup $T$ such that $\mathrm{F}(T)=\mathrm{F}$ and $S \subsetneq T$. Arguing as in the proof of the Proposition 4.2 , we deduce that $\mathcal{R}(S) \neq \emptyset$. 


\section{P-semigroups with maximal embedding dimension}

It is well known (see for instance Proposition 2.10 of [4]) that if $S$ is a numerical semigroup then $e(S) \leq \mathrm{m}(S)$. A numerical semigroup $S$ has maximal embedding dimension (MED-semigroup) if e $(S)=\mathrm{m}(S)$. The following result is a consequence of Proposition I.2.9 from [1].

Lemma 5.1. Let $S$ be a numerical semigroup. Then $S$ is a MED-semigroup if and only if $S^{\prime}=\{s-\mathrm{m}(S) \mid s \in S \backslash\{0\}\}$ is a numerical semigroup.

The following result relates $S$ and $S^{\prime}$ in the case where $S$ is also a P-semigroup.

Proposition 5.2. Let $S$ be a MED-semigroup. Then $S$ is a P-semigroup if and only if $S^{\prime}=\{s-\mathrm{m}(S) \mid s \in S \backslash\{0\}\}$ is a P-semigroup and $\mathrm{m}(S)$ is odd.

Proof. Necessity. By Lemma 2.1, we know that if $S$ is a P-semigroup, then $\mathrm{m}(S)$ is odd. From Lemma 5.1 we know that $S^{\prime}$ is a numerical semigroup. Now we see that $S^{\prime}$ is a P-semigroup. It is clear that if $S=\left\{s_{0}=0, s_{1}, s_{2}, \ldots\right\}$ then $S^{\prime}=\left\{0, s_{2}-s_{1}, s_{3}-s_{1}, \ldots\right\}$. As $s_{1}+s_{2}$ is odd, then $0+s_{2}-s_{1}$ is also odd. If $i \in \mathbb{N} \backslash\{0,1\}$, then $s_{i}-s_{1}+s_{i+1}-s_{1}=$ $s_{i}+s_{i+1}-2 s_{1}$ is odd. Hence $S^{\prime}$ is a P-semigroup.

Sufficiency. It is clear that if $S=\left\{s_{0}=0, s_{1}, s_{2}, \ldots\right\}$ then $S^{\prime}=\left\{0, s_{2}-s_{1}, s_{3}-s_{1}, \ldots\right\}$. As $s_{1}=\mathrm{m}(S)$, then $s_{0}+s_{1}$ is odd. Applying that $S^{\prime}$ is a P-semigroup we have that $0+s_{2}-s_{1}$ is odd and so, $s_{1}+s_{2}$ is odd. If $i \in \mathbb{N} \backslash\{0,1\}$ then $s_{i}+s_{i+1}$ is odd because $s_{i}-s_{1}+s_{i+1}-s_{1}$ is odd. Therefore $S$ is a P-semigroup.

The following result is a consequence of Lemma 5.1.

Lemma 5.3. Let $S$ be a numerical semigroup and $x \in S \backslash\{0\}$. Then $S(x)=(\{x\}+S) \cup\{0\}$ is a MED-semigroup with multiplicity x. Moreover, every MED-semigroup has this form.

A PMED-semigroup is a MED-semigroup that in addition is a P-semigroup.

Proposition 5.4. Let $S$ be a P-semigroup and $x \in S$ such that $x$ is odd. Then $S(x)=$ $(\{x\}+S) \cup\{0\}$ is a PMED-semigroup. Moreover, every PMED-semigroup has this form.

Proof. By Lemma 5.3, we know that $S(x)$ is a MED-semigroup. It is clear that $S=$ $\{s-m(S(x)) \mid s \in S(x) \backslash\{0\}\}$. Therefore, applying Proposition 5.2, we have that $S(x)$ is a P-semigroup. Thus, $S(x)$ is a PMED-semigroup.

Let $T$ be a PMED-semigroup. Then, using Proposition 5.2, we deduce that $S=\{t-$ $\mathrm{m}(T) \mid t \in T \backslash\{0\}\}$ is a P-semigroup and $\mathrm{m}(T)$ is odd. Thus $T=(\{\mathrm{m}(T)\}+S) \cup\{0\}$ with $\mathrm{m}(T) \in S$ and $\mathrm{m}(T)$ odd.

The following result can be deduced from [3, Proposition 9].

Proposition 5.5. Let $S$ be a P-semigroup, $x \in S \backslash\{0,1\}$ and $S(x)=(\{x\}+S) \cup\{0\}$. Then $\mathrm{F}(S(x))=\mathrm{F}(S)+x$ and $\mathrm{g}(S(x))=\mathrm{g}(S)+x-1$.

Next we illustrate the previous results with an example.

Example 5.6. Let $S=\langle 5,8,9\rangle=\{0,5,8,9,10,13, \rightarrow\}$. It is clear that $S$ is a P-semigroup, $\mathrm{F}(S)=12$ and $\mathrm{g}(S)=8$. As $9 \in S$ and 9 is odd, then applying Proposition 5.4 and Proposition 5.5, we have that $S(9)=(\{9\}+S) \cup\{0\}=\{0,9,14,17,18,19,22, \rightarrow\}$ is a PMED-semigroup with Frobenius number $12+9=21$ and genus $8+9-1=16$.

Acknowledgment. The authors wish to thank the referee for his valuable comments and suggestions. 


\section{References}

[1] V. Barucci, D.E. Dobbs and M. Fontana, Maximality Properties in Numerical Semigroups and Applications to One-Dimensional Analitycally Irreducible Local Domains, Memoirs Amer. Math. Soc. 598, 1997.

[2] M.A. Moreno-Frías and J.C. Rosales, Perfect numerical semigroups, Turkish J. Math. 43, 1742-1754, 2019.

[3] J.C. Rosales, Principal ideals of numerical semigroups, Bull. Belg. Math. Soc. 10, $1-15,2003$.

[4] J.C. Rosales and P.A. García-Sánchez, Numerical Semigroups, Developments in Mathematics, 20, Springer, New York, 2009.

[5] J.C. Rosales, P.A. García-Sánchez, J.I. García-García and J.A. Jiménez-Madrid, The oversemigroups of a numerical semigroup, Semigroup Forum, 67, 145-158, 2003. 